\title{
Economic Burden of Metastatic Ovarian Cancer in a Commercially Insured Population: A Retrospective Cohort Analysis
}

\author{
Lauren Chin, PharmD, MS; Ryan N. Hansen, PharmD, PhD; and Josh J. Carlson, MPH, PhD
}

\begin{abstract}
BACKGROUND: Ovarian cancer is the tenth most common type of cancer and the fifth leading cause of cancer death among females in the United States. The majority of incident ovarian cancer cases are diagnosed in individuals aged $<65$ years, but limited evidence exists regarding the economic burden of ovarian cancer in this age group.
\end{abstract}

OBJECTIVES: To (a) estimate the annual all-cause direct total cost of metastatic ovarian cancer and (b) compare it to the cost of individuals without cancer in the working age commercially insured U.S. population.

METHODS: We conducted a retrospective cohort analysis using the IBM MarketScan Commercial Database. Patients were included if they met the following criteria: $\geq 1$ medical claim with a secondary malignancy diagnosis in the primary position between January 1, 2011, and December 31, 2015 (earliest date of diagnosis defined as the index date); aged $\geq 18$ years on the index date; $\geq 12$ months of continuous enrollment before the index date; $\geq 1$ month of continuous enrollment after the index date; and $\geq 1$ inpatient medical claim or $\geq 2$ outpatient medical claims $\geq 30$ days apart, with an ovarian cancer diagnosis in any claim position within 60 days before or 30 days after the index date. Patients were excluded if they had $\geq 1$ medical claim with a cancer diagnosis except for ovarian cancer in any claim position during the 12-month pre-index period. Controls were randomly selected and matched to metastatic ovarian cancer patients based on age, region, index date, number of months of continuous enrollment after the index date, and propensity score. Annual all-cause direct total costs and ovarian cancer-related direct total costs were estimated and compared for each cohort by using the Kaplan-Meier sample average technique to account for censoring after the index date.

RESULTS: 2,991 metastatic ovarian cancer patients and 2,991 matched controls were included in this study. Patients in the metastatic ovarian cancer cohort had a mean (SD) age of 54.4 (8.5) years, and controls had a mean (SD) age of $54.2(8.4)$ years. The mean $(95 \% \mathrm{Cl})$ annual all-cause total costs in the 12-month post-index period were $\$ 140,124$ $(\$ 134,025-\$ 146,267)$ for metastatic ovarian cancer patients and $\$ 35,161$ $(\$ 31,338-\$ 39,529)$ for controls; the resulting mean $(95 \% \mathrm{Cl})$ difference in annual all-cause total costs was $\$ 104,964$ (\$99,732-\$110,042). In comparison with the annual all-cause total costs, the mean $(95 \% \mathrm{Cl})$ annual ovarian cancer-related total costs in the 12-month post-index period were $\$ 86,971$ (\$82,349-\$91,508) for metastatic ovarian cancer patients and $\$ 0(\$ 0-\$ 0)$ for controls.

CONCLUSIONS: Working age patients with metastatic ovarian cancer have significantly higher costs compared with those without cancer. These findings contribute to the understanding of the burden of illness in a patient population where limited evidence currently exists on the economic consequences of the disease.

J Manag Care Spec Pharm. 2020;26(8):962-70

Copyright $\odot 2020$, Academy of Managed Care Pharmacy. All rights reserved.

\section{What is already known about this subject}

Previous studies have evaluated the cost of ovarian cancer among Medicare patients.

One study of patients aged $\geq 65$ years with advanced ovarian cancer, for example, estimated that their annual direct total costs ranged from $\$ 85,987$ to $\$ 89,149$.

\section{What this study adds}

This is the first study on the economic burden of metastatic ovarian cancer in commercially insured females aged $<65$ years.

The economic burden of metastatic ovarian cancer patients is substantial and significantly greater than that of patients without cancer.

$\Lambda$ mong women in the United States, ovarian cancer is the tenth most common type of cancer and the fifth leading cause of cancer death. ${ }^{1}$ In 2020, it is estimated that there will be 21,750 new cases and 13,940 deaths attributable to ovarian cancer in the United States. ${ }^{2}$ Fifty-nine percent of ovarian cancer patients have distant disease at diagnosis, and the corresponding 5-year relative survival rate is $29.2 \% .^{2}$

There are several known risk factors for ovarian cancer, including obesity, nulliparity, and the use of unopposed estrogen therapy. ${ }^{3}$ Women of advanced age and those who are carriers of mutations in BRCA1/2 are also at an increased risk of developing the disease. ${ }^{4}$ In the general population, the average age of onset is 63 years, and the lifetime risk is approximately $1.3 \% .^{2}$ Conversely, BRCA1 and BRCA2 carriers have an estimated lifetime risk of $44 \%$ and $17 \%$, respectively, by age 80 years and a mean age of onset of 50.0 and 54.5 years, respectively. ${ }^{4,5}$ Based on data from the Surveillance, Epidemiology, and End Results program from 2011 to 2016, $54.2 \%$ of patients were younger than age 65 years at the time of diagnosis. ${ }^{2}$

For the majority of newly diagnosed cases, first-line treatment generally involves surgery and/or interval debulking followed by chemotherapy, depending on surgical candidacy but without regard to stage at initial presentation. ${ }^{6}$ Newer targeted therapies (e.g., olaparib, niraparib, and rucaparib) have improved the treatment landscape for individuals with susceptible tumor types, but the associated costs remain disproportionately high relative to the marginal improvement in 
progression-free survival provided by these treatment agents. ${ }^{\top}$ Recent studies have evaluated the initial costs (e.g., 12-month costs after diagnosis; first-line surgery and/or chemotherapy costs after diagnosis) of ovarian cancer and costs incurred among the Medicare population. ${ }^{89}$ However, given that the majority of cases are diagnosed at an advanced stage, and more than half of incident cases are diagnosed at under age 65 years, it is important to establish the cost of the disease in a relevant epidemiological context.

To the best of our knowledge, no study has evaluated the economic burden of metastatic ovarian cancer in only working age patients. The primary objectives of this study were therefore to estimate the annual all-cause direct total cost of metastatic ovarian cancer and to determine the incremental cost of metastatic ovarian cancer patients compared with the corresponding costs of patients without cancer in the working age commercially insured U.S. population. The secondary objective of this study was to estimate the annual ovarian cancer-related direct total cost among metastatic ovarian cancer patients.

\section{Methods}

\section{Data Source}

This retrospective study used administrative claims data from the IBM MarketScan Commercial Database from January 1, 2010, through December 31, 2016. The MarketScan database provides longitudinal and cross-sectional views of health care practices and costs for the working population and their dependents and aggregates paid claims data for several million individuals annually from employers and health plans across the United States. ${ }^{10,11}$ All patient records are deidentified, and a unique identifier links each patient's associated medical claims, pharmacy claims, and enrollment information. Since this study used only deidentified patient records, pursuant to the Health Insurance Portability and Accountability Act of 1996, this study did not require institutional review board waiver nor approval.

\section{Study Design}

This study was a retrospective cohort analysis that evaluated allcause direct costs and ovarian cancer-related direct costs among patients with metastatic ovarian cancer in the 12-month period following diagnosis of metastases. Eligible patients were identified through MarketScan from January 1, 2011, through December 31, 2015, and selected based on predefined inclusion criteria.

\section{Study Sample}

Patients in the metastatic ovarian cancer cohort were first identified based on having $\geq 1$ medical claim with a secondary malignancy diagnosis (International Classification of Diseases, Ninth/Tenth Revision, Clinical Modification [ICD-9-CM] codes 196.xx-198.xx; [ICD-10-CM] codes C77.xx-C79.xx) in the primary position from January 1, 2011, through December 31,
2015 (index period); the date of the earliest claim with a secondary malignancy diagnosis (i.e., metastases) in the index period was defined as the index date. Additionally, patients must have also been female on the index date; aged $\geq 18$ years on the index date; had $\geq 12$ months of continuous enrollment before the index date; had $\geq 1$ month of continuous enrollment after the index date; and had $\geq 1$ inpatient medical claim or $\geq 2$ outpatient medical claims $\geq 30$ days apart with an ovarian cancer diagnosis (ICD-9-CM codes 183.xx; ICD-10-CM codes C56.xx) in any claim position within 60 days before or 30 days after the index date. Patients were excluded if they had a medical claim with a cancer diagnosis (ICD-9-CM codes 140.xx-239.xx; ICD-10-CM codes C00.xx-D49.xx) except for ovarian cancer in any claim position during the 12 -month pre-index period. The last selection criterion was used in order to focus the analysis on incident metastatic ovarian cancer patients.

Patients in the control cohort were first identified based on having $\geq 1$ medical claim during the index period using a random sample of patients derived from 25,000 random inpatient claims and 25,000 random outpatient claims from each index year (2011, 2012, 2013, 2014, and 2015; the index date was defined as the date of the earliest claim for a patient within a given year). Patients must have also been female on the index date; aged $\geq 18$ years on the index date; and had $\geq 12$ months of continuous enrollment before the index date. Patients were excluded if they had $\geq 1$ medical claim with a cancer diagnosis in any position in the 12 months before the index date, on the index date, or in the 12 months after the index date.

After the selection of patients for the control cohort, we derived a propensity score for each metastatic ovarian cancer patient $(n=2,991)$ and control $(n=67,914)$ using a logistic regression model in which metastatic ovarian cancer status was regressed on the following baseline (i.e., 12-month pre-index period, inclusive of the index date) clinical and demographic characteristics: age (continuous); region (Northeast, North Central, South, West, and unknown); plan type (basic/ major medical, comprehensive, exclusive provider organization [EPO], health maintenance organization [HMO], point of service [POS], preferred provider organization [PPO], POS with capitation, consumer-driven health plan [CDHP], highdeductible health plan [HDHP], and unknown); polycystic ovarian syndrome (yes, no); hypertension (yes, no); ischemic heart disease (yes, no); diabetes mellitus (yes, no); endometriosis (yes, no); asthma (yes, no); autoimmune diseases (yes, no); depression (yes, no); anxiety (yes, no); osteoporosis (yes, no); kidney disease (yes, no); liver disease (yes, no); gallbladder disease (yes, no); and pain (yes, no).

Each patient had a predicted probability, ranging from 0 through 1 , of being a metastatic ovarian cancer patient based on her individual demographic and clinical characteristics.

Note that the Deyo-Charlson Comorbidity Index (CCI) score was not included in the logistic regression model for 
propensity score estimation, since it was highly correlated with the outcome (i.e., metastatic ovarian cancer patients would receive $\geq 6 \mathrm{CCI}$ score points for having a diagnosis of cancer and/or metastases, while all controls were not allowed to have any diagnosis of cancer based on the selection criteria). A randomly selected subset of control patients were then matched to the metastatic ovarian cancer patients in a 1:1 manner based on age ( \pm 5 years), region, index date ( \pm 30 days), number of months of continuous enrollment after the index date, and logit of the propensity score $( \pm 0.2$ of the standard deviation [SD]). All matching variables were weighted equally; controls were therefore matched exactly to metastatic ovarian cancer patients based on the aforementioned parameters.

\section{Subgroup with $\geq 12$ Months of Continuous Enrollment After the Index Date}

Subgroup analyses were conducted in which only patients with $\geq 12$ months of continuous enrollment after the index date were included. Therefore, all patients were required to have survived and remain enrolled throughout the 12-month follow-up period due to having continuous enrollment throughout this post-index period. The purpose of this subgroup analysis was to evaluate the outcomes of interest in a patient sample that was not lost to follow-up within 12 months after the index date.

\section{Study Variables}

Baseline demographic characteristics, including age (continuous; 18-34, 35-44, 45-54, and 55-65 years); region (Northeast, North Central, South, West, and unknown); and plan type (basic/major medical, comprehensive, EPO, HMO, PPO, POS with capitation, CDHP, HDHP, and unknown) were measured on the index date for the metastatic ovarian cancer patients and controls. Baseline clinical characteristics were also measured in the 12-month pre-index period, inclusive of the index date, for each cohort. The CCI score (continuous; 0, 1, 2-4, and $\geq 5$ ), along with the presence of general comorbid conditions (i.e., polycystic ovarian syndrome, hypertension, ischemic heart disease, diabetes mellitus, endometriosis, asthma, autoimmune diseases, depression, anxiety, osteoporosis, kidney disease, liver disease, gallbladder disease, and pain ${ }^{12,13}$ ) were evaluated for each patient.

Outcomes of interest included annual all-cause direct total cost (i.e., cost due to any reason; summation of patient-paid and plan-paid medical and pharmacy costs) and annual ovarian cancer-related direct total cost (i.e., cost based on claims with an ovarian cancer diagnosis in any position or pharmacy claims for treatments that can be used for ovarian cancer [rucaparib, olaparib, niraparib, anastrozole, tamoxifen citrate, letrozole, exemestane, leuprolide acetate, megestrol acetate, and pazopanib]), which were measured in the 12-month post-index period for both cohorts. Although the control cohort was not expected to have any ovarian cancer-related costs, such costs were evaluated to confirm our expectations and provide complete data on both cohorts.

Costs were presented as unadjusted costs and also by subcomponents (e.g., medical costs and pharmacy costs); medical costs were also further presented as outpatient costs and inpatient costs. Additionally, the incremental costs for metastatic ovarian cancer patients versus controls were presented for each cost variable. Note that no adjusted costs (i.e., costs taking into account controlling for potential confounders) were presented, since patient characteristics for metastatic ovarian cancer patients and controls were balanced as a result of matching.

\section{Statistical Analyses}

All baseline demographic and clinical characteristics were evaluated descriptively for each cohort. Continuous variables were summarized as means and SDs, and categorical variables were summarized as frequencies and percentages. Standardized differences were also calculated for baseline characteristics to assess the extent to which both cohorts were balanced based on propensity score matching.

To evaluate differences in annual costs between the metastatic ovarian cancer patients and controls and account for varying lengths of post-index follow-up time, we applied the Kaplan-Meier sample average (KMSA) estimator to assess the annual costs per cohort in the presence of censoring after the index date. ${ }^{14-16}$ The per-cohort costs for each of the 12 monthly intervals after the index date were first estimated by averaging the monthly costs for each interval for each cohort. For example, post-index month 1 costs for metastatic ovarian cancer patients were estimated by averaging the month 1 costs for metastatic ovarian cancer patients with $\geq 1$ month of continuous enrollment after the index date; note that all patients with $\geq 1$ month of continuous enrollment after the index date were alive at the beginning of the 1 month after the index date. Post-index month 2 costs for the same cohort were then calculated by averaging the costs in post-index month 2 for patients with at $\geq 2$ months of continuous enrollment after the index date. Each monthly interval was associated with an equal or lesser value for the denominator in subsequent months, and the denominator for the average monthly costs varied based on month.

The KMSA method involves multiplying the probability of surviving to the start of each time interval (i.e., post-index month) by the costs incurred during the corresponding time interval conditional on surviving to the start of that interval. Using the study data, Kaplan-Meier survival curves were constructed using data on censoring (i.e., no additional continuous enrollment data after a certain post-index month without evidence of death) and death (an inpatient claim with a discharge diagnosis of death). In order to assess the annual all-cause costs and ovarian cancer-related costs for each cohort while accounting for censoring and death after the index date, the 


\section{FIGURE 1 Study Cohort Selection}

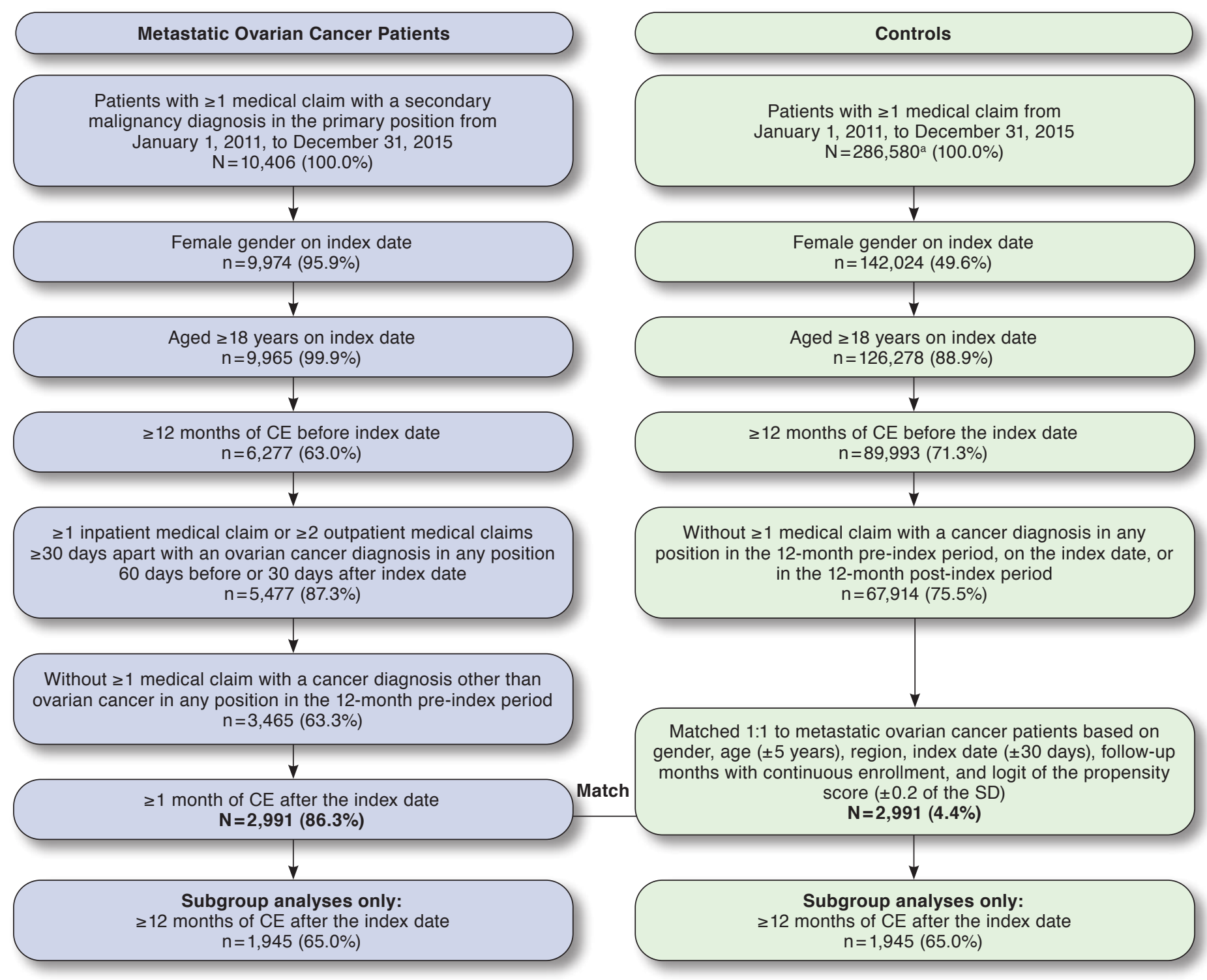

aBased on 25,000 random inpatient claims and 25,000 random outpatient claims each year $C E=$ continuous enrollment $; S D=$ standard deviation

average monthly cost for each cohort (12 months total) were summed to calculate the point estimates for the annual costs.

For the annual post-index costs for each cohort, 95\% confidence intervals (CIs) were calculated by using a bootstrap approach with 1,000 iterations in which 1,000 pairs of matched metastatic ovarian cancer patients and controls were sampled with equal probability and without replacement (i.e., simple random sampling) from the original cohorts. For each bootstrap sample, the annual cost was calculated via summation of monthly costs (the KMSA estimator was applied to the mean cost at each monthly interval). A bootstrap approach was used, since individual annual costs could not be calculated for each patient due to varying lengths of post-index follow-up time.

Among the subgroup of metastatic ovarian cancer patients and controls with $\geq 12$ months of continuous enrollment after the index date, annual all-cause costs and ovarian cancerrelated costs (based on the 12 months after the index date) 


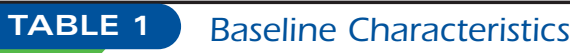

\begin{tabular}{|c|c|c|c|c|c|}
\hline \multirow[b]{2}{*}{ Age (years), mean (SD) } & \multicolumn{2}{|c|}{$\begin{array}{c}\text { Metastatic } \\
\text { Ovarian Cancer } \\
(\mathbf{n}=2,991)\end{array}$} & \multicolumn{2}{|c|}{$\begin{array}{l}\text { Controls } \\
(\mathrm{n}=2,991)\end{array}$} & \multirow{2}{*}{\begin{tabular}{|c}
$\begin{array}{c}\text { Standardized } \\
\text { Difference }\end{array}$ \\
0.02 \\
\end{tabular}} \\
\hline & 54.4 & $(8.5)$ & 54.2 & (8.4) & \\
\hline \multicolumn{6}{|l|}{ Age group (years), n (\%) } \\
\hline $18-34$ & 91 & $(3.0)$ & 91 & $(3.0)$ & 0 \\
\hline $35-44$ & 260 & $(8.7)$ & 262 & $(8.8)$ & 0 \\
\hline $45-54$ & 882 & $(29.5)$ & 886 & $(29.6)$ & 0 \\
\hline $55-65$ & 1,758 & $(58.8)$ & 1,752 & $(58.6)$ & 0 \\
\hline \multicolumn{6}{|l|}{ Region, n (\%) } \\
\hline Northeast & 613 & $(20.5)$ & 613 & $(20.5)$ & 0 \\
\hline North Central & 727 & $(24.3)$ & 727 & $(24.3)$ & 0 \\
\hline South & 1,032 & $(34.5)$ & 1,032 & $(34.5)$ & 0 \\
\hline West & 578 & $(19.3)$ & 578 & $(19.3)$ & 0 \\
\hline Unknown & 41 & $(1.4)$ & 41 & $(1.4)$ & 0 \\
\hline \multicolumn{6}{|l|}{ Plan type, n (\%) } \\
\hline Comprehensive & 107 & (3.6) & 99 & (3.3) & 0.01 \\
\hline EPO & 32 & $(1.1)$ & 35 & $(1.2)$ & -0.01 \\
\hline $\mathrm{HMO}$ & 275 & $(9.2)$ & 295 & (9.9) & -0.02 \\
\hline POS & 177 & $(5.9)$ & 175 & (5.9) & 0 \\
\hline $\mathrm{PPO}$ & 1,412 & $(47.2)$ & 1,484 & $(49.6)$ & -0.05 \\
\hline POS with capitation & 19 & $(0.6)$ & 13 & $(0.4)$ & 0.03 \\
\hline CDHP & 195 & $(6.5)$ & 169 & $(5.7)$ & 0.04 \\
\hline HDHP & 94 & (3.1) & 61 & $(2.0)$ & 0.07 \\
\hline Unknown & 680 & $(22.7)$ & 660 & $(22.1)$ & 0.02 \\
\hline CCI score, mean (SD) & 6.7 & $(3.1)$ & 0.8 & (1.3) & 2.50 \\
\hline \multicolumn{6}{|l|}{ CCI score, $\mathrm{n}(\%)$} \\
\hline 0 & 0 & $(0.0)$ & 1,757 & $(58.7)$ & -1.69 \\
\hline 1 & 0 & $(0.0)$ & 721 & $(24.1)$ & -0.80 \\
\hline $2-4$ & 931 & $(31.1)$ & 428 & $(14.3)$ & 0.41 \\
\hline$\geq 5$ & 2,060 & $(68.9)$ & 85 & $(2.8)$ & 1.90 \\
\hline \multicolumn{6}{|l|}{ Comorbidities, n (\%) } \\
\hline $\begin{array}{l}\text { Polycystic ovarian } \\
\text { syndrome }\end{array}$ & 16 & $(0.5)$ & 11 & $(0.4)$ & 0.02 \\
\hline Hypertension & 1,074 & $(35.9)$ & 1,059 & $(35.4)$ & 0.01 \\
\hline Ischemic heart disease & 144 & $(4.8)$ & 132 & $(4.4)$ & 0.02 \\
\hline Diabetes mellitus & 384 & $(12.8)$ & 371 & $(12.4)$ & 0.01 \\
\hline Endometriosis & 3 & $(0.1)$ & 2 & $(0.1)$ & 0.01 \\
\hline Asthma & 42 & (1.4) & 46 & (1.5) & -0.01 \\
\hline Autoimmune diseases & 30 & $(1.0)$ & 37 & $(1.2)$ & -0.02 \\
\hline Depression & 5 & $(0.2)$ & 4 & $(0.1)$ & 0.01 \\
\hline Anxiety & 44 & $(1.5)$ & 47 & (1.6) & -0.01 \\
\hline Osteoporosis & 13 & $(0.4)$ & 17 & $(0.6)$ & -0.02 \\
\hline Kidney disease & 35 & $(1.2)$ & 37 & $(1.2)$ & -0.02 \\
\hline Liver disease & 25 & $(0.8)$ & 20 & $(0.7)$ & 0.02 \\
\hline Gallbladder disease & 7 & $(0.2)$ & 7 & $(0.2)$ & 0 \\
\hline Pain & 19 & $(0.6)$ & 19 & $(0.6)$ & 0 \\
\hline $\begin{array}{l}\begin{array}{l}\text { Follow-up time } \\
\text { (months), mean (SD) }\end{array} \\
\end{array}$ & 10.12 & (3.0) & 10.12 & (3.0) & 0 \\
\hline
\end{tabular}

CCI = Deyo-Charlson Comorbidity Index; $C D H P=$ consumer-driven health plan; $E P O=$ exclusive provider organization; HDHP = high-deductible health plan; $\mathrm{HMO}=$ health maintenance organization; $\mathrm{POS}=$ point of service; $\mathrm{PPO}=$ preferred provider organization; $S D=$ standard deviation were summarized as means and 95\% CIs and compared using Student's t-tests.

\section{Results}

\section{Baseline Characteristics}

A total of 2,991 metastatic ovarian cancer patients and 2,991 matched controls were included in this study (Figure 1) Demographic and clinical characteristics were not significantly different between both cohorts, with the exception of the CCI score. Patients in the metastatic ovarian cancer cohort had a mean (SD) age of 54.4 (8.5) years, and controls had a mean (SD) age of 54.2 (8.4) years; $58.8 \%$ and $58.6 \%$ of metastatic ovarian cancer patients and controls, respectively, were aged 55 to 65 years. All patients were female and matched on region, with the South and North Central regions as the most represented regions (34.5\% and $24.3 \%$, respectively, for both cohorts). The majority of patients in both cohorts had insurance coverage through a PPO plan type (47.2\% and $49.6 \%$ for metastatic ovarian cancer patients and controls, respectively; Table 1).

The mean (SD) CCI score was 6.7 (3.1) for metastatic ovarian cancer patients and 0.8 (1.3) for controls; note that all metastatic ovarian cancer patients had $\geq 6$ CCI points due to having metastases on the index date. In relation to comorbidities, both cohorts were balanced, and the most prevalent conditions were hypertension, diabetes mellitus, and ischemic heart disease. All other comorbidities of interest had a prevalence of less than $2 \%$ (asthma, autoimmune diseases, anxiety, and kidney disease) or less than $1 \%$ (polycystic ovarian syndrome, endometriosis, depression, osteoporosis, liver disease, gallbladder disease, and pain; Table 1).

\section{Annual Costs: Primary Analyses}

The mean (95\% CI) annual all-cause total costs in the 12 -month post-index period were $\$ 140,124$ ( $\$ 134,025$ $\$ 146,267)$ for the metastatic ovarian cancer patients and $\$ 35,161(\$ 31,338-\$ 39,529)$ for the controls; the resulting mean (95\% CI) difference in annual all-cause total costs was $\$ 104,964$ $(\$ 99,732-\$ 110,042)$. The mean $(95 \% \mathrm{CI})$ annual ovarian cancerrelated total costs in the 12-month post-index period were $\$ 86,971(\$ 82,349-\$ 91,508)$ for the metastatic ovarian cancer patients and $\$ 0(\$ 0)$ for the controls. The majority of all-cause and ovarian cancer-related total costs in the follow-up period were driven by medical costs for both cohorts. Pharmacy costs contributed only a small percentage to all-cause and ovarian cancer-related total costs for both cohorts (Table 2).

\section{Annual Costs: Subgroup Analyses}

When excluding patients without $\geq 12$ months of continuous enrollment after the index date, 1,945 patients remained in the metastatic ovarian cancer and control cohorts (Figure 1). Baseline characteristics, with the exception of CCI score, were not significantly different between cohorts (Table 3). 


\begin{tabular}{|c|c|c|c|c|c|c|}
\hline & Metastatic Ovarian Cancer & \multicolumn{2}{|r|}{ Controls } & \multicolumn{2}{|c|}{$\begin{array}{c}\text { Difference (Metastatic Ovarian } \\
\text { Cancer vs. Controls) }\end{array}$} & $P$ Value $^{\mathrm{a}}$ \\
\hline \multicolumn{7}{|c|}{ Annual all-cause costs, mean $(95 \% \mathrm{CI})$} \\
\hline Total costs & $\$ 140,124 \quad(\$ 134,025-\$ 146,267)$ & $\$ 35,161$ & $(\$ 31,338-\$ 39,529)$ & $\$ 104,964$ & $(\$ 99,732-\$ 110,042)$ & $<0.05$ \\
\hline Medical costs & $\$ 136,412 \quad(\$ 130,392-\$ 142,482)$ & $\$ 33,072$ & $(\$ 29,247-\$ 37,380)$ & $\$ 103,340$ & $(\$ 98,205-\$ 108,351)$ & $<0.05$ \\
\hline Inpatient costs & $\$ 67,735 \quad(\$ 63,686-\$ 71,870)$ & $\$ 23,142$ & $(\$ 19,713-\$ 26,979)$ & $\$ 44,594$ & $(\$ 40,631-\$ 48,524)$ & $<0.05$ \\
\hline Outpatient costs & $\$ 68,677 \quad(\$ 65,567-\$ 72,104)$ & $\$ 9,931$ & $(\$ 8,915-\$ 11,137)$ & $\$ 58,746$ & $(\$ 56,193-\$ 61,208)$ & $<0.05$ \\
\hline Pharmacy costs & $(\$ 3,296-\$ 4,119)$ & $\$ 2,088$ & $(\$ 1,815-\$ 2,399)$ & $\$ 1,624$ & $(\$ 1,254-\$ 1,964)$ & $<0.05$ \\
\hline \multicolumn{7}{|c|}{ Annual ovarian cancer-related costs, mean (95\% CI) } \\
\hline Total costs & $\$ 86,971 \quad(\$ 82,349-\$ 91,508)$ & $\$ 0$ & $(\$ 0)$ & $\$ 86,971$ & $(\$ 83,728-\$ 90,171)$ & $<0.05$ \\
\hline Medical costs & $\$ 86,965 \quad(\$ 82,344-\$ 91,505)$ & $\$ 0$ & $(\$ 0)$ & $\$ 86,965$ & $(\$ 83,722-\$ 90,166)$ & $<0.05$ \\
\hline Inpatient costs & $\$ 42,964 \quad(\$ 40,083-\$ 45,964)$ & $\$ 0$ & $(\$ 0)$ & $\$ 42,964$ & $(\$ 40,821-\$ 45,081)$ & $<0.05$ \\
\hline Outpatient costs & $\$ 44,001 \quad(\$ 41,152-\$ 46,693)$ & $\$ 0$ & $(\$ 0)$ & $\$ 44,001$ & $(\$ 42,037-\$ 45,950)$ & $<0.05$ \\
\hline Pharmacy costs & $(\$ 2-\$ 10)$ & $\$ 0$ & $(\$ 0)$ & $\$ 5$ & $(\$ 2-\$ 8)$ & $<0.05$ \\
\hline
\end{tabular}

aStudent's t-tests were used to compare differences between cohorts.

The mean $(95 \% \mathrm{CI})$ annual all-cause total costs in the 12 -month post-index period were $\$ 165,619$ (\$160,363$\$ 170,875)$ for the metastatic ovarian cancer patients and $\$ 36,625$ (\$33,437-\$39,813) for the controls; the resulting difference in annual all-cause total costs was a mean (95\% CI) of $\$ 128,994$ ( $\$ 122,846-\$ 135,142)$. The mean $(95 \% \mathrm{CI}$ ) annual ovarian cancer-related total costs in the follow-up period were $\$ 105,477$ (\$101,382-\$109,572) for the metastatic ovarian cancer patients and $\$ 0(\$ 0)$ for the controls. The majority of all-cause and ovarian cancer-related costs in the follow-up period were driven by medical costs for both cohorts, similar to that in the primary analyses. Pharmacy costs contributed only a small percentage to all-cause costs and ovarian cancer-related total costs for both cohorts (Table 4).

\section{Discussion}

Ovarian cancer remains a substantial clinical and economic burden in the United States. Our primary objectives were to estimate the annual all-cause direct total costs in metastatic ovarian cancer patients and to compare the costs of metastatic ovarian cancer patients with costs in patients without cancer in the working age commercially insured U.S. population. We found that the mean annual all-cause total cost of patients with metastatic ovarian cancer was approximately $\$ 140,000$ in the primary analysis, with the majority of costs related to ovarian cancer. When compared with controls that were matched on baseline demographic and clinical characteristics, metastatic ovarian cancer patients incurred approximately $\$ 105,000$ greater all-cause total costs compared with controls.

To our knowledge, this is the first study to describe the annual costs of metastatic ovarian cancer in working age patients. Previous studies have evaluated the costs of ovarian cancer but have been limited to older data or patients aged $\geq 65$ years. ${ }^{8,9}$ This study demonstrates the economic burden of an understudied patient population that may be inherently more complex or incur higher costs because of earlier disease onset and more aggressive treatment approaches used in this population. One study that evaluated patients aged $\geq 65$ years with advanced ovarian cancer reported mean annual direct total costs ranging from $\$ 85,987$ to $\$ 89,149 .{ }^{9}$ A recent study also found that costs across different types of cancer were higher for patients aged $<65$ years compared with those aged $\geq 65$ years. ${ }^{17}$ Future research focused on this younger patient population will provide greater context for our results and better understanding of the drivers of high costs associated with this younger age group.

All-cause costs were driven primarily by medical costs. This is consistent with findings from a previous study that found that inpatient and outpatient services accounted for nearly all of the $\$ 93,632$ in mean total expenditures during the 12-month postoperative period for patients newly diagnosed with ovarian cancer. ${ }^{8}$ Although we did not evaluate medical costs by type, the major cost driver may be chemotherapy costs. This is supported by a study on the initial phase of care in ovarian cancer patients, which demonstrated that medical costs are primarily driven by chemotherapy costs. ${ }^{9}$ At diagnosis, $59 \%$ of patients have distal metastases, and $21 \%$ of patients have regional metastases (i.e., spread to lymph nodes), so the majority of costs in a nonrestrictive sample of patients with ovarian cancer are expected to be for patients with metastatic disease. ${ }^{2}$

Pharmacy costs were much lower in comparison, which is representative of the limited number of treatments available for metastases that can be self-administered by patients (e.g., oral poly ADP-ribose polymerase inhibitors). Patients with metastatic ovarian cancer are typically treated with platinum-based chemotherapy (e.g., paclitaxel/cisplatin, paclitaxel/carboplatin, docetaxel/carboplatin, carboplatin/liposomal doxorubicin, paclitaxel/carboplatin/bevacizumab, and 


\begin{tabular}{|c|c|c|c|}
\hline & $\begin{array}{c}\text { Metastatic } \\
\text { Ovarian } \\
\text { Cancer } \\
(\mathrm{n}=1,945)\end{array}$ & $\begin{array}{l}\text { Controls } \\
(\mathrm{n}=1,945)\end{array}$ & $\begin{array}{l}\text { Standardized } \\
\text { Difference }\end{array}$ \\
\hline Age (years), mean (SD) & $54.5 \quad(8.2)$ & $54.3 \quad(8.1)$ & 0.02 \\
\hline \multicolumn{4}{|l|}{ Age group (years), n (\%) } \\
\hline $18-34$ & $53 \quad(2.7)$ & $54 \quad(2.8)$ & 0 \\
\hline $35-44$ & $150 \quad(7.7)$ & $151 \quad(7.8)$ & 0 \\
\hline $45-54$ & $580(29.8)$ & $586(30.1)$ & -0.01 \\
\hline $55-65$ & $1,162 \quad(59.7)$ & $1,154(59.3)$ & 0.01 \\
\hline \multicolumn{4}{|l|}{ Region, n (\%) } \\
\hline Northeast & $377(19.4)$ & $377(19.4)$ & 0 \\
\hline North Central & $470(24.2)$ & $470(24.2)$ & 0 \\
\hline South & $669(34.4)$ & $669(34.4)$ & 0 \\
\hline West & $395(20.3)$ & $395(20.3)$ & 0 \\
\hline Unknown & $34 \quad(1.8)$ & $34 \quad(1.8)$ & 0 \\
\hline \multicolumn{4}{|l|}{ Plan type, n (\%) } \\
\hline Comprehensive & $84 \quad(4.3)$ & $81 \quad(4.2)$ & 0.01 \\
\hline EPO & $23 \quad(1.2)$ & $26 \quad(1.3)$ & -0.01 \\
\hline $\mathrm{HMO}$ & $223(11.5)$ & $228(11.7)$ & -0.01 \\
\hline POS & $134 \quad(6.9)$ & $125 \quad(6.4)$ & 0.02 \\
\hline $\mathrm{PPO}$ & $1,023(52.6)$ & $1,089(56.0)$ & -0.07 \\
\hline POS with capitation & $14 \quad(0.7)$ & $9 \quad(0.5)$ & 0.03 \\
\hline CDHP & $127 \quad(6.5)$ & $130 \quad(6.7)$ & -0.01 \\
\hline HDHP & $69 \quad(3.6)$ & $45 \quad(2.3)$ & 0.07 \\
\hline Unknown & $248(12.8)$ & $212(10.9)$ & 0.06 \\
\hline CCI score, mean (SD) & $6.6 \quad(3.0)$ & $0.7 \quad(1.3)$ & 2.52 \\
\hline \multicolumn{4}{|l|}{ CCI score, $\mathrm{n}(\%)$} \\
\hline 0 & $\begin{array}{ll}0 & (0) \\
\end{array}$ & $1,169(60.1)$ & -1.74 \\
\hline 1 & $0 \quad(0)$ & $467(24.0)$ & -0.79 \\
\hline $2-4$ & $612(31.5)$ & $261(13.4)$ & 0.44 \\
\hline$\geq 5$ & $1,333(68.5)$ & $48 \quad(2.5)$ & 1.91 \\
\hline \multicolumn{4}{|l|}{ Comorbidities, n (\%) } \\
\hline $\begin{array}{l}\text { Polycystic ovarian } \\
\text { syndrome }\end{array}$ & $10 \quad(0.5)$ & $8 \quad(0.4)$ & 0.02 \\
\hline Hypertension & $680(35.0)$ & $665(34.2)$ & 0.02 \\
\hline Ischemic heart disease & $98 \quad(5.0)$ & $82 \quad(4.2)$ & 0.04 \\
\hline Diabetes mellitus & $239(12.3)$ & $239(12.3)$ & 0 \\
\hline Endometriosis & $2 \quad(0.1)$ & $1 \quad(0.1)$ & 0.02 \\
\hline Asthma & $18 \quad(0.9)$ & $26 \quad(1.3)$ & -0.04 \\
\hline Autoimmune diseases & $13 \quad(0.7)$ & $16 \quad(0.8)$ & -0.02 \\
\hline Depression & $4 \quad(0.2)$ & $1 \quad(0.1)$ & 0.04 \\
\hline Anxiety & $28 \quad(1.4)$ & $20 \quad(1.0)$ & 0.04 \\
\hline Osteoporosis & $8 \quad(0.4)$ & $9 \quad(0.5)$ & -0.01 \\
\hline Kidney disease & $18 \quad(0.9)$ & $21 \quad(1.1)$ & -0.02 \\
\hline Liver disease & $14 \quad(0.7)$ & $13 \quad(0.7)$ & 0.01 \\
\hline Gallbladder disease & $3 \quad(0.2)$ & $3 \quad(0.2)$ & 0 \\
\hline Pain & $11 \quad(0.6)$ & $8 \quad(0.4)$ & 0.02 \\
\hline
\end{tabular}

$C C I=$ Deyo-Charlson Comorbidity Index; $C D H P=$ consumer-driven health plan; $E P O=$ exclusive provider organization; $H D H P=$ high-deductible health plan; $H M O=$ health maintenance organization; $P O S=$ point of service; $P P O=$ preferred provider organization; $S D=$ standard deviation. paclitaxel/carboplatin/bevacizumab) and completion surgery (as indicated by tumor response and potential resectability), which would not involve medication dispensing from a pharmacy or other pharmacy services (e.g., medication therapy management). ${ }^{6}$ With the approval of additional oral therapies after 2016 (e.g., niraparib and rucaparib), however, future research may demonstrate higher pharmacy costs. Additionally, metastatic ovarian cancer patients incurred substantially higher costs unrelated to ovarian cancer compared with controls. This may be due to the effect of ovarian cancer on other comorbidities or potential undercoding of ovarian cancer in claims data.

Costs of controls in this study may appear relatively high, even for patients without cancer, and may not be representative of the typical patient without cancer. Per-person national health expenditures in 2014, for example, were estimated to be $\$ 7,153$ per working age individual and $\$ 19,098$ for those aged $\geq 65$ years. $^{18}$ The results of this study are most likely because controls were matched to metastatic ovarian cancer patients on age and propensity score, which led to balanced demographic and clinical characteristics between both cohorts, with the exception of cancer status. Nonetheless, the primary objective of this study was to determine the incremental annual all-cause total cost due to metastatic ovarian cancer. Matching of controls to the metastatic ovarian cancer patients helped achieve this objective by controlling for potential confounders.

When comparing the costs of metastatic ovarian cancer patients with $\geq 1$ month of continuous enrollment after the index date versus those of metastatic ovarian cancer patients with $\geq 12$ months of continuous enrollment after the index date, the mean annual all-cause total costs of the former were approximately $15 \%$ less; results were similar for controls. One potential reason why costs were higher among metastatic ovarian cancer patients with $\geq 12$ months of continuous enrollment after the index date is that patients who survived longer were able to incur greater costs. In the event that costs were lower for these patients, which may potentially occur in other datasets, it may be due to patients incurring high costs only in the months leading up to death from ovarian cancer and primarily after 12 months.

\section{Limitations}

This study has several limitations associated with the use of retrospective claims data. First, patients may have been diagnosed with ovarian cancer more than 12 months before the index date but may have gone into remission; patients with recurrent ovarian cancer may thus have been included in this analysis, which intended only to include incident cases.

Second, the lack of complete data on mortality in the MarketScan database precludes an accurate assessment of 
TABLE 4 Annual Follow-up Costs for Subgroup with $\geq 12$ Months of Follow-up

\begin{tabular}{|c|c|c|c|c|c|}
\hline & $\begin{array}{l}\text { Metastatic Ovarian Cancer } \\
\qquad(\mathrm{n}=1,945)\end{array}$ & \multicolumn{2}{|c|}{ Controls $(n=1,945)$} & $\begin{array}{l}\text { Difference (Metastatic Ovarian } \\
\text { Cancer vs. Controls) }\end{array}$ & $P$ Value $^{\mathrm{a}}$ \\
\hline \multicolumn{6}{|c|}{ Annual all-cause costs, mean $(95 \% \mathrm{CI})$} \\
\hline Total costs & $\$ 165,619 \quad(\$ 160,363-\$ 170,875)$ & $\$ 36,625$ & $(\$ 33,437-\$ 39,813)$ & $\$ 128,994 \quad(\$ 122,846-\$ 135,142)$ & $<0.05$ \\
\hline Medical costs & $\$ 160,515 \quad(\$ 155,358-\$ 165,672)$ & $\$ 33,708$ & $(\$ 30,594-\$ 36,822)$ & $\$ 126,807 \quad(\$ 120,783-\$ 132,831)$ & $<0.05$ \\
\hline Inpatient costs & $(\$ 65,230-\$ 72,066)$ & $\$ 21,434$ & $(\$ 18,789-\$ 24,079)$ & $(\$ 42,892-\$ 51,536)$ & $<0.05$ \\
\hline Outpatient costs & $(\$ 88,451-\$ 95,283)$ & $\$ 12,274$ & $(\$ 11,297-\$ 13,250)$ & $(\$ 76,041-\$ 83,146)$ & $<0.05$ \\
\hline Pharmacy costs & $(\$ 4,632-\$ 5,575)$ & $\$ 2,917$ & $(\$ 2,589-\$ 3,245)$ & $(\$ 1,612-\$ 2,761)$ & $<0.05$ \\
\hline \multicolumn{6}{|c|}{ Annual ovarian cancer-related costs, mean (95\% CI) } \\
\hline Total costs & $\$ 105,477 \quad(\$ 101,382-\$ 109,572)$ & $\$ 0$ & $(\$ 0)$ & $\$ 105,477 \quad(\$ 101,382-\$ 109,572)$ & $<0.05$ \\
\hline Medical costs & $\$ 105,470 \quad(\$ 101,375-\$ 109,565)$ & $\$ 0$ & $(\$ 0)$ & $\$ 105,470 \quad(\$ 101,375-\$ 109,565)$ & $<0.05$ \\
\hline Inpatient costs & $(\$ 42,145-\$ 46,879)$ & $\$ 0$ & $(\$ 0)$ & $(\$ 42,145-\$ 46,879)$ & $<0.05$ \\
\hline Outpatient costs & $(\$ 58,019-\$ 63,898)$ & $\$ 0$ & $(\$ 0)$ & $(\$ 58,019-\$ 63,898)$ & $<0.05$ \\
\hline Pharmacy costs & $(\$ 3-\$ 10)$ & $\$ 0$ & $(\$ 0)$ & $(\$ 3-\$ 10)$ & $<0.05$ \\
\hline
\end{tabular}

annual costs after an incident diagnosis of metastatic ovarian cancer. Therefore, we used the KMSA technique to estimate costs and account for patients that were lost to follow-up.

Third, there is limited data surrounding relevant confounders in the context of metastatic ovarian cancer. We accounted for comorbidities in this study based on the literature; however, more studies need to be conducted in order to assess the strength of association between the included comorbidities and metastatic ovarian cancer. Fourth, the study sample was limited to patients aged $\leq 65$ years with commercial insurance, and findings may not be generalizable to older patients or those covered primarily by public health plans.

Finally, not all patients included in this study may have been working, since dependents of working individuals (e.g., young adults and spouses) are also included in the MarketScan database. This may have explained the upper age limit of 65 years, but a more plausible explanation is that many of these patients were aged $<65$ years at the beginning of the year when they were enrolled in a commercial insurance plan (hence their inclusion in this database).

\section{Conclusions}

Working age patients with metastatic ovarian cancer have significantly higher costs compared with those without cancer. Given that the majority of ovarian cancer patients are diagnosed at age $<65$ years, these findings contribute to the understanding of the burden of illness in a patient population where little evidence currently exists regarding the economic consequences of the disease.

\section{Authors}

LAUREN CHIN, PharmD, MS, Formulary Resources, Mercer Island, Washington. RYAN N. HANSEN, PharmD, PhD, and JOSH J. CARLSON, MPH, PhD, The Comparative Health Outcomes, Policy, and Economics Institute, University of Washington, Seattle.

AUTHOR CORRESPONDENCE: Lauren Chin, PharmD, MS, Clinical Pharmacist, Formulary Resources, Mercer Island, WA 98040. Tel.: 425.223.8766; Email: lchin@uw.edu.

\section{DISCLOSURES}

No outside funding supported this study. The authors have nothing to disclose.

This study was presented at the 2019 International Society for Pharmacoeconomics and Outcomes Research Annual Meeting; May 18-22, 2019; New Orleans, LA.

\section{REFERENCES}

1. Centers for Disease Control and Prevention. Leading cancer cases and deaths, all races/ethnicities, male and female, 2017. 2018. Available at: https://gis.cdc.gov/Cancer/USCS/DataViz.html. Accessed May 12, 2019

2. National Cancer Institute. SEER cancer stat facts: ovarian cancer. 2018 Available at: https://seer.cancer.gov/statfacts/html/ovary.html. Accessed June 24, 2020

3. American Cancer Society. Ovarian cancer risk factors. 2018. Available at: https://www.cancer.org/cancer/ovarian-cancer/causes-risks-prevention/riskfactors.html. Accessed June 24, 2020.

4. Kuchenbaecker KB, Hopper JL, Barnes DR, et al. Risks of breast, ovarian, and contralateral breast cancer for BRCAl and BRCA2 mutation carriers. JAMA. 2017;317(23):2402-16

5. Rebbeck TR, Mitra N, Wan F, et al. Association of type and location of BRCA1 and BRCA2 mutations with risk of breast and ovarian cancer. JAMA. 2015;313(13):1347-61. 
6. National Comprehensive Cancer Network. Ovarian cancer including fallopian tube cancer and primary peritoneal cancer (Version 2.2018). 2018 Available at: https://www.nccn.org/professionals/physician_gls/pdf/ovarian. pdf. Accessed June 24, 2020.

7. Liu AY, Walsh CS, Holschneider CH, Sinno AK. A cost-effectiveness analysis of three PARP inhibitors for maintenance therapy in platinumsensitive recurrent ovarian cancer [abstract]. Gynecol Oncol. 2017;147(1):196.

8. Bercow AS, Chen L, Chatterjee S, et al. Cost of care for the initial management of ovarian cancer. Obstet Gynecol. 2017;130(6):1269-75.

9. Urban RR, He H, Alfonso-Cristancho R, Hardesty MM, Goff BA. The cost of initial care for Medicare patients with advanced ovarian cancer. J Natl Compr Canc Netw. 2016;14(4):429-37.

10. IBM Watson Health. IBM MarketScan Research Databases. 2018. Available at: https://www.ibm.com/downloads/cas/4QD5ADRL. Accessed June 24, 2020.

11. Truven Health Analytics. The Truven Health MarketScan Databases for Health Services Researchers. IBM Watson Health. 2017. Available at: https:// truvenhealth.com/portals/0/assets/2017_MarketScan_Databases_Health_ Services_Researchers.pdf. Accessed June 24, 2020.

12. Minlikeeva AN, Freudenheim JL, Eng KH et al. History of comorbidities and survival of ovarian cancer patients, results from the Ovarian Cancer Association Consortium. Cancer Epidemiol Biomarkers Prev. 2017;26(9):1470-73.
13. Harris HR, Terry KL. Polycystic ovary syndrome and risk of endometrial, ovarian, and breast cancer: a systematic review. Fertility Res Pract. 2016;2:14. Published online December 5, 2016. Available at: https:// www.ncbi.nlm.nih.gov/pmc/articles/PMC5424400/. Accessed June 24, 2020.

14. Wijeysundera HC, Wang X, Tomlinson G, Ko, DT, Krahn MD. Techniques for estimating health care costs with censored data: an overview for the health services researcher. Clinicoecon Outcomes Res. 2012;4:145-55.

15. Lin DY. Linear regression analysis of censored medical costs. Biostatistics. 2000;1(1):35-47. Available at: http://biostatistics.oxfordjournals.org/ content/1/1/35.long. Accessed June 24, 2020.

16. Lin DY, Feuer EJ, Etzioni R, Wax Y. Estimating medical costs from incomplete follow-up data. Biometrics. 1997;53(2):419-34. Available at: http:// dlin.web.unc.edu/files/2013/04/LinEA97.pdf. Accessed June 24, 2020.

17. Banegas MP, Yabroff KR, O'Keeffe-Rosetti MC, et al. Medical care costs associated with cancer in integrated delivery systems. J Natl Compr Canc Netw. 2018;16(4):402-10.

18. Centers for Medicare \& Medicaid Services. NHE fact sheet. April 26, 2019. Available at: https://www.cms.gov/research-statistics-dataand-systems/statistics-trends-and-reports/nationalhealthexpenddata/ nhe-fact-sheet.html. Accessed June 24, 2020. 